\title{
Micro-imaging of buried layers and interfaces in ultrathin films by X-ray reflectivity
}

Jinxing Jiang (蒋金星 ), Keiichi Hirano (平野馨一), and Kenji Sakurai (桜井健次)

Citation: Journal of Applied Physics 120, 115301 (2016); doi: 10.1063/1.4962311

View online: $\mathrm{https}: / /$ doi.org/10.1063/1.4962311

View Table of Contents: http://aip.scitation.org/toc/jap/120/11

Published by the American Institute of Physics

\section{Articles you may be interested in}

$\mathrm{X}$-ray reflectivity imager with $15 \mathrm{~W}$ power $\mathrm{X}$-ray source

Review of Scientific Instruments 87, 093709 (2016); 10.1063/1.4962408

Perspective: Interface generation of spin-orbit torques

Journal of Applied Physics 120, 180901 (2016); 10.1063/1.4967391

Delocalization of vortex in $\mathrm{SmBa}_{2} \mathrm{Cu}_{3} \mathrm{O}_{7-\delta}$ superconducting films with $\mathrm{BaHfO}_{3}$ nano-rods Journal of Applied Physics 120, 103902 (2016); 10.1063/1.4962398

Directional thermal emission control by coupling between guided mode resonances and tunable plasmons in multilayered graphene

Journal of Applied Physics 120, 163105 (2016); 10.1063/1.4966577

Mechanical properties of hydrogenated electron-irradiated graphene Journal of Applied Physics 120, 124301 (2016); 10.1063/1.4962716

The smooth transition from field emission to a self-sustained plasma in microscale electrode gaps at atmospheric pressure

Journal of Applied Physics 119, 223301 (2016); 10.1063/1.4953648

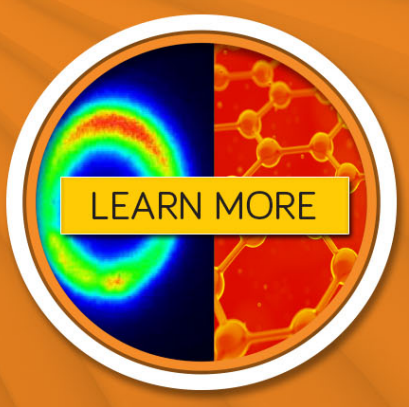




\title{
Micro-imaging of buried layers and interfaces in ultrathin films by X-ray reflectivity
}

\author{
Jinxing Jiang (蒋金星), ${ }^{1,2}$ Keiichi Hirano (平野馨一), ${ }^{3}$ and Kenji Sakurai (桜井健次) ${ }^{2,1}$ \\ ${ }^{1}$ University of Tsukuba, 1-1-1, Tennodai, Tsukuba, Ibaraki 305-0006, Japan \\ ${ }^{2}$ National Institute for Materials Science, 1-2-1, Sengen, Tsukuba, Ibaraki 305-0047, Japan \\ ${ }^{3}$ Photon Factory, High Energy Accelerator Research Organization, KEK, 1-1, Oho, Tsukuba, \\ Ibaraki 305-0087, Japan
}

(Received 23 June 2016; accepted 22 August 2016; published online 15 September 2016)

\begin{abstract}
$\mathrm{X}$-ray reflectivity is a promising technique for characterizing buried layers and interfaces in ultrathin films because of its ability to probe the electron density profile along the depth in a nondestructive manner. While routine X-ray reflectivity assumes the in-plane uniformity of the sample to be measured, it is also quite important to see buried inhomogeneous/patterned layers and interfaces. The present paper describes the addition of spatial resolution and imaging capability to an $\mathrm{X}$-ray reflectivity technique to visualize surfaces and buried interfaces. To visualize quite wide viewing area size quickly, the image reconstruction scheme has been employed instead of the scanning of microbeam. Though the mathematics is quite close to X-ray computer tomography, the technique gives the image contrast caused by the difference in reflectivity at each in-plane point in the thin film sample. By choosing a grazing angle, the image gives inhomogeneity of X-ray reflectivity at the specific wavevector transfer. With a collimated monochromatic synchrotron X-ray beam of $0.05 \mathrm{~mm}(\mathrm{H}) \times 8 \mathrm{~mm}(\mathrm{~V})$, the intensity profiles of X-ray reflection projections have been taken at many different in-plane rotation angles, from $0^{\circ}$ to $180^{\circ}$. We have succeeded in visualizing buried layers and interfaces of the $8 \mathrm{~mm}$ dia area with the spatial resolution of better than $20 \mu \mathrm{m}$. Because of the brilliance of synchrotron radiation, the typical measuring time is shorter than $1 \mathrm{~min}$. Three analytical cases have been discussed: (i) imaging of a buried layer and an interface covered by a protection layer, (ii) distinguishing different local parts of different thicknesses in an ultrathin film, and (iii) selective imaging of a specific metal in the thin film form. Published by AIP Publishing. [http://dx.doi.org/10.1063/1.4962311]
\end{abstract}

\section{INTRODUCTION}

$\mathrm{X}$-ray reflectivity (XR) is sensitive to slight structural changes along the depth of layered thin film materials in the order of sub-nanometers or sub-angstroms. Conventional $\mathrm{X}$-ray reflectivity gives "average" information over quite a large area, typically $\mathrm{mm}^{2}-\mathrm{cm}^{2}$. When the local structures along the depth are not uniform and there exist several different layered structures depending on the location of the sample, it is necessary to extend X-ray reflectivity's capability in the micro area. ${ }^{1}$ Even where the layered structure is almost uniform, usually the local heterogeneity presents the key in solving problems. In modern research, there have been a lot of unsolved but interesting scientific problems in inhomogeneous thin films, and thus, it is extremely important to have an imaging technique to search for key points. Invented surfaces and interfaces imaging techniques include $\mathrm{X}$-ray reflection phase-contrast full field microscopy by Fenter et al., ${ }^{2-5}$ a lensless X-ray imaging technique in reflection geometry applying post scattering phase coding by Roy et al., ${ }^{6}$ a three-dimensional coherent X-ray surface scattering imaging technique by Sun et al., ${ }^{7}$ and a ptychographic X-ray imaging technique on crystal truncation rod by Zhu et al. ${ }^{8}$ Although these techniques are promising and show possibilities for specific applications, many of these techniques require sophisticated optics, while others rely on coherent $\mathrm{X}$-rays or special samples. We invented a complementary imaging method called X-ray reflectivity imaging (XRI) that is devoted to visualization of buried layers and interfaces in ultrathin films. XRI is realized by introducing an image reconstruction scheme to X-ray reflectivity. Compared with the methods mentioned above, it shows several advantages such as: (1) an optics-free technique, no need of X-ray optics such as Fresnel Zone plates or Kirkpatrick-Baez mirrors (KB) to focus X-rays; (2) a user-friendly and easily accessible system, no reliance on coherence of X-rays, while the experiment could be performed even in a laboratory; (3) a large scale viewing area of the sample $[10 \mathrm{~mm} \times 10 \mathrm{~mm}$, works well both for crystal and non-crystal thin films] without perspective effects due to the image reconstruction scheme; (4) tunable imaging depth fashion. The image contains the information depending on the $\mathrm{X}$-rays penetration depth, which could be tuned by the incident angle or X-ray energy. The X-ray reflectivity imaging experiments have been performed, and the capabilities in the laboratory have been demonstrated in parallel beam geometry ${ }^{9,10}$ with a spatial resolution of $1.6 \mathrm{~mm}$ and in fan beam geometry ${ }^{11}$ with a spatial resolution of $0.18 \mathrm{~mm}$. A synchrotron radiation source provides brilliant $\mathrm{X}$-rays up to 10 decades more brilliant than a laboratory X-ray source. In addition, since the distance between the source (insertion device) and the experimental hutch is quite far (typically $20 \mathrm{~m}$ ), the angular resolution is much better than that of laboratory cases. Introducing synchrotron radiation to reflectivity imaging is promising. In the 


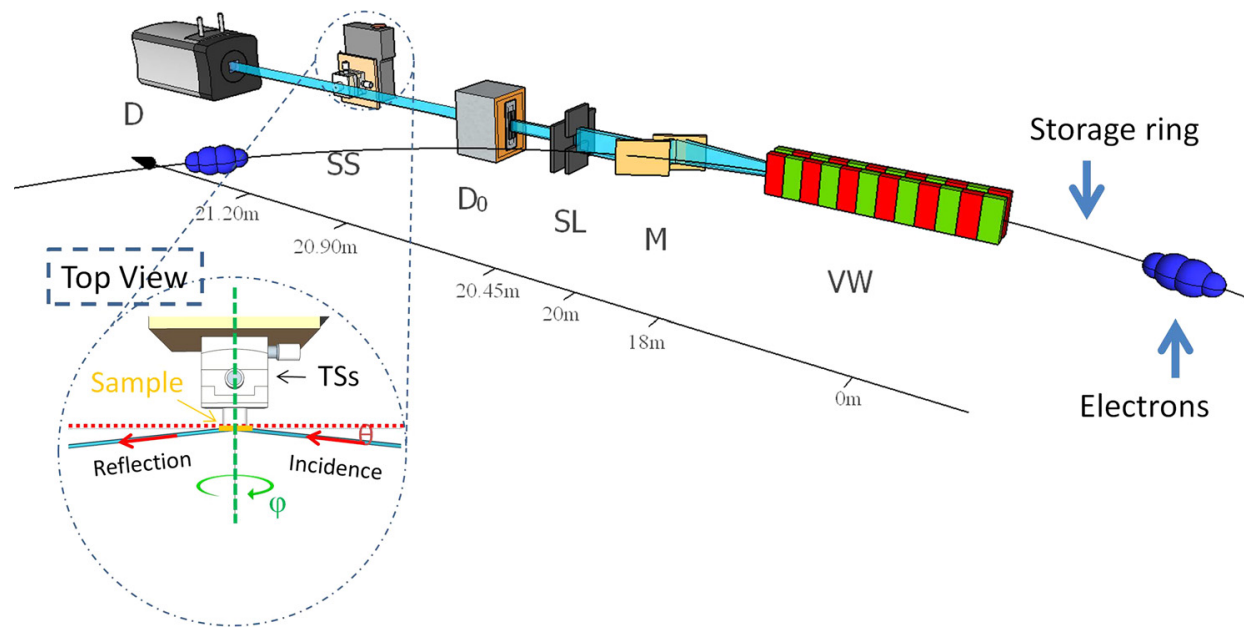

FIG. 1. Perspective view of the experimental set-up of X-ray reflectivity imaging technique. Storage ring: orbit of free electron bunches; VW: Vertical Wiggler to generate synchrotron radiation; M: double crystal Monochromator; SL: $2 \mathrm{D}$ slit $[1 \mathrm{~mm}(\mathrm{H}) \times 8 \mathrm{~mm}(\mathrm{~V})]$; $\mathrm{D}_{0}$ : Ionization Chamber which records incident X-ray intensity, with a horizontal slit $(0.05 \mathrm{~mm}, \mathrm{H})$ attached on the top window; SS: sample stage standing on a high precision goniometer; D: CCD camera with a scintillation film suitable for X-ray detection. Inset is the enlarged image of SS in the top view: TSs: tilting stages to align the sample to be perpendicular to the in-plane rotational axis. $\theta$ is the grazing incident angle and the exit angle between X-rays and sample surface. The thick dashed line in the top view shows the in-plane rotational axis.

present paper, we present the first results of X-ray reflectivity imaging of buried layers and interfaces in ultrathin films by using synchrotron radiation achieving a spatial resolution of $20 \mu \mathrm{m}$.

\section{EXPERIMENTAL}

We performed the experiment at Beamline $14 \mathrm{~B},{ }^{12}$ Photon Factory, Tsukuba, Japan. The experimental set-up is shown in Fig. 1. An insertion superconducting vertical wiggler provided white X-ray beam polarized in the vertical plane. The white and vertically polarized beam was monochromatized by a fixed-exit double-crystal $\mathrm{Si}(111)$ monochromator, with an energy resolution of $\sim 10^{-4} .16 \mathrm{keV}$ $\mathrm{X}$-rays were chosen because the energy is at around the peak position of the power spectrum. The primary beam size was $7 \mathrm{~mm}(\mathrm{H}) \times 15 \mathrm{~mm}(\mathrm{~V})$. A primary collimating 4D slit was set at the furthest upstream side of the experiment hutch, which was $20 \mathrm{~m}$ away from the wiggler source, to collimate the beam to $1 \mathrm{~mm}(\mathrm{H}) \times 8 \mathrm{~mm}(\mathrm{~V})$. The X-ray intensity was being monitored all the time during the experiment by an ionization chamber (IC) set $0.45 \mathrm{~m}$ behind the 4D slit. In front of the entrance window of the IC, a fixed width $(50 \mu \mathrm{m}$, $\mathrm{H})$ slit was attached to further cut the horizontal width of the beam, thus the final incident beam size was $0.05 \mathrm{~mm}(\mathrm{H})$ $\times 8 \mathrm{~mm}(\mathrm{~V})$. The sample stage, set $0.45 \mathrm{~m}$ downward from the IC, was composed of an in-plane rotational motor, two mutually orthogonal manually micro-tilting stages, a vacuum chuck, and a high precision $\theta / 2 \theta$ goniometer (not shown in Fig. 1). The in-plane rotational motor can scan the in-plane angle from $0^{\circ}$ to $180^{\circ}$ with a positioning accuracy of $0.1^{\circ}$. Micro-tilting stages were used to adjust the sample to tilt to the proper angle, thereby ensuring that the sample surface plane was perpendicular to the rotational axis of the in-plane motor. This procedure secured that the incidence was always the same during the in-plane rotational scanning. The vacuum chuck was a specific sample holder designed to hold the flat samples vertically. The components were set on the goniometer, with which the grazing incident angle could be precisely adjusted. At the downstream side, a CCD camera equipped with a scintillation film and capillary optics was set $0.30 \mathrm{~m}$ behind the sample as the position sensitive detector, which was used to record reflection projections and was set on the $2 \theta$ arm of the goniometer. Further instrumental details are described elsewhere. ${ }^{11}$

This technique was inspired by the merits of X-ray reflectivity's high sensitivity for analyzing thin films in depth and the urgent demand for an imaging technique to visualize heterogeneities in thin films. X-ray reflectivity is measured by $\theta / 2 \theta$ scan, where $\theta$ is the grazing incident angle ${ }^{13}$ and related to the wavevector transfer $\mathrm{Q}_{\mathrm{z}}$ (normalized by X-rays wavelength $\lambda$ ) by

$$
Q_{z}=\frac{4 \pi \sin \theta}{\lambda} .
$$

Howbeit in case of XRI, by performing an in-plane rotational scan at a specific incident angle (or related specific $Q_{z}$ ), we reconstructed a reflectivity image at the corresponding wavevector transfer $\mathrm{Q}_{\mathrm{z}}$, and such a contrast is strongly correlated to the in-depth structure of thin films. Unlike the ordinary X-ray computer tomography in transmission geometry, by employing grazing incidence reflection geometry XRI achieves extremely high sensitivity in depth such as sub nm scale as the same as XR. The signal retrieved from the sample is not the absorption coefficient, but the surfaces and interfaces scattering (reflectivity) from the sample. The reconstructed image is the in-plane spatial mapping (in real space) of X-ray reflectivity at a specific wavevector transfer $\mathrm{Q}_{\mathrm{z}}$ (in momentum space). Although this technique gives a $2 \mathrm{D}$ image, it also includes in-depth information (as $\mathrm{X}$-rays' penetration depth is strongly correlated to $\mathrm{Q}_{\mathrm{z}}$, as will be discussed in Section III). By proper sampling at each in-plane rotational scan (2D image) and at each wavevector transfer $\mathrm{Q}_{\mathrm{z}}$ (different reflectivity contrast), we could extract the differences 
inside the ultrathin film at specific depths and help solve the critical problems in such materials systems.

In order to obtain enough information to reconstruct the reflectivity images, we recorded many $1 \mathrm{D}$ reflection projections sampled at in-plane rotational angles evenly from $0^{\circ}$ to $180^{\circ}$ at fixed grazing incidence. The reflection projections were recorded by the CCD camera as many TIF image files. (Note that the X-rays' footprints on the CCD camera are not perfectly $1 \mathrm{D}$ projections but narrow rectangles as the incident X-rays horizontally have the width of $50 \mu \mathrm{m}$.) In order to efficiently handle many TIF images, we use many Python opensource libraries designed for scientific computing such as numpy, matplotlib, and Tkinter. For the data reduction and processing, we prepared Python codes to read each TIF file, specify the area of interests, and integrate the reflection rectangles into $1 \mathrm{D}$ projection with the batch mode compatibility. Mathematically, the reflection projection is the integral reflection intensity profile along the X-ray forward direction according to the Radon transform ${ }^{14}$

$$
p_{\varphi}(r)=\int_{-\infty}^{\infty} f(r \cos \varphi-z \sin \varphi, r \sin \varphi+z \cos \varphi) d z
$$

where $\varphi$ is the in-plane angle, $\mathrm{r}$ is the projection positions (experimental pixel number on the $\mathrm{CCD}$ ), $\mathrm{z}$ is the $\mathrm{X}$-rays forward direction, $\mathrm{f}(\mathrm{x}, \mathrm{y})$ is the $2 \mathrm{D}$ reflectivity image, and $\mathrm{p}_{\varphi}(\mathrm{r})$ is the $1 \mathrm{D}$ reflection projection profile at in-plane angle $\varphi$.

For the X-ray reflectivity image reconstruction process, we prepared the software based on the convolution backprojection algorithm using Object-Pascal coded GUI. According to the Fourier Slice theorem ${ }^{15}$ and the convolution theorem, we could reconstruct an XRI image from 1D projections by back projecting all convolved reflection projections at different in-plane angles:

$$
f(x, y)=\int_{0}^{\pi} g_{\varphi}(x \cos \varphi+y \sin \varphi) d \varphi,
$$

(a) Reflection Projection $\mathrm{R}\left(Q_{z}\right)$ Map

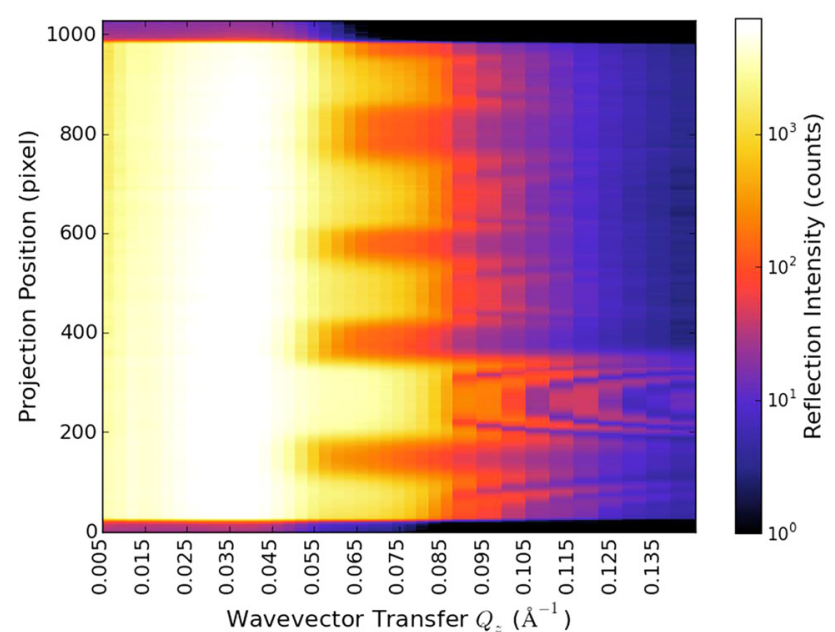

where $\mathrm{f}(\mathrm{x}, \mathrm{y})$ is the $2 \mathrm{D}$ reflectivity image, which in other word is the reflectivity distribution at the local position labeled by $(\mathbf{x}, \mathbf{y})$. The image is discretized into $x-y$ grid, and interpolation is inevitable noticing we need to back project the data at different in-plane angles. Here the simplest linear interpolation is employed. $\mathrm{g}_{\varphi}(\mathrm{r})$ is the convolved projection at a specific in-plane angle

$$
g_{\varphi}(r)=h(r) * p_{\varphi}(r),
$$

in which $\mathrm{P}_{\varphi}(\mathrm{r})$ is the $1 \mathrm{D}$ reflection projection at a specific inplane angle $\varphi, *$ indicates the sign of convolution, and $\mathrm{h}(\mathrm{r})$ is the convolution kernel. [It is mathematically the same as to multiply the projections with the Ram-Lak filter function H(r) in frequency domain according to the convolution theorem.]

$$
h(r)=\frac{f_{c}}{\pi r} \sin \left(2 \pi r f_{c}\right)-\frac{1}{(\pi r)^{2}} \sin ^{2}\left(\pi r f_{c}\right),
$$

where $f_{c}$ is the cutoff frequency of the Ram-Lak filter in Fourier space. According to the Nyquist sampling theorem, ${ }^{16}$ this value was set as 0.5 or smaller when conducting the image reconstruction.

\section{RESULTS AND DISCUSSION}

As discussed in Section II, an XRI image is a spatial mapping of $\mathrm{X}$-ray reflectivity at a specific $\mathrm{Q}_{\mathrm{z}}$. In order to decide $\mathrm{Q}_{\mathrm{z}}$ of interest, we firstly measured $\mathrm{X}$-ray reflectivity by scanning grazing incident angle/exit angle $(\theta / 2 \theta)$ at a fixed in-plane rotational angle $\varphi$ before performing the $\mathrm{X}$-ray reflectivity imaging measurement. In X-ray reflectivity, X-ray reflection projections at each incident angle were recorded by the CCD camera and the reflection projections were plotted other than $\mathrm{Q}_{\mathrm{z}}$, as shown in Figs. 2(a), 4(a), and 6(a). In the X-ray reflectivity imaging experiment, grazing incident angle was fixed, and reflection projections at different in-plane angles were recorded by the CCD camera. This

(b)

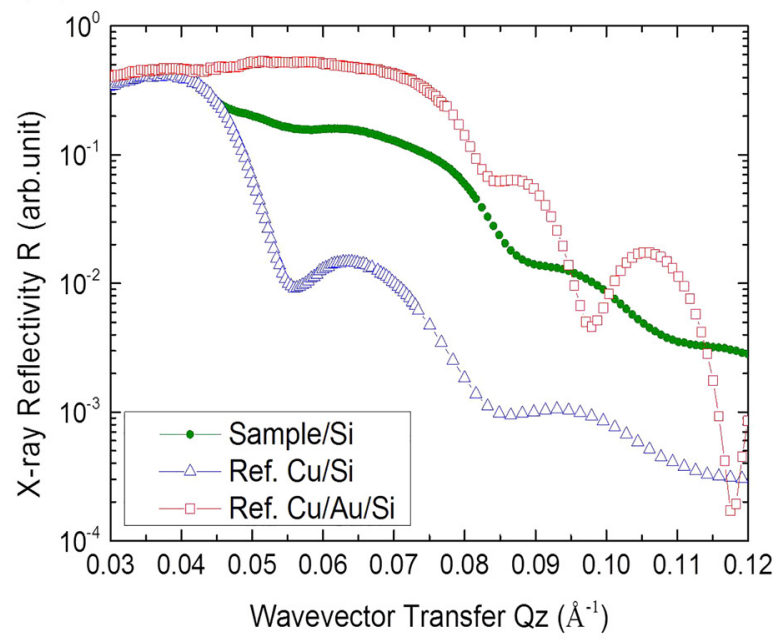

FIG. 2. X-ray reflection profiles of the copper/gold(pattern)/Si sample and its reference samples. (a) Local reflectivity profiles of the patterned sample measured at the SR facility as the reflection projection map of the wavevector transfer $\mathrm{Q}_{\mathrm{z}}$. The scanning step for the measurement is $\Delta \mathrm{Q}_{\mathrm{z}}=0.003 \AA^{-1}$, and the data are plotted in logarithmic color scale. (b) Ordinary X-ray reflectivity profiles of the patterned sample (circle), the reference uniform copper/gold/Si sample (rectangle), and the reference uniform copper/Si sample (triangle) measured by a laboratory X-ray reflectometer. 

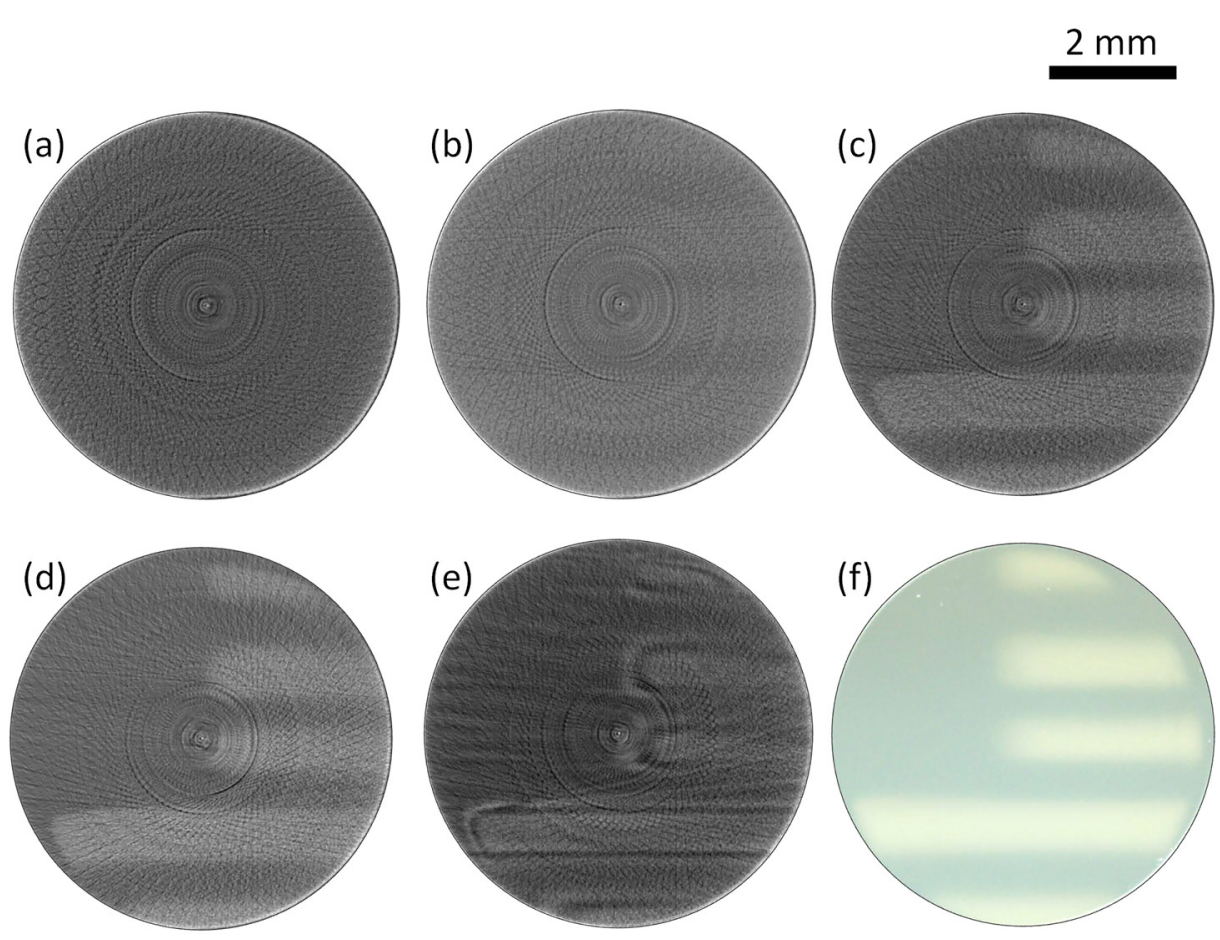

FIG. 3. Reconstructed X-ray reflectivity images of the copper/gold(pattern)/ Si sample at different wavevector transfers. Parameters for image reconstruction: 36 views, cutoff frequency for Ram-Lak filter: 0.2. (a) $\mathrm{Q}_{\mathrm{z}}=0.024 \AA^{-1}$; (b) $\mathrm{Q}_{\mathrm{z}}=0.049 \AA^{-1}$; (c) $\mathrm{Q}_{\mathrm{z}}=0.057 \AA^{-1}$; (d) $\mathrm{Q}_{\mathrm{z}}=0.073 \AA^{-1}$; (e) $\mathrm{Q}_{\mathrm{z}}=0.098 \AA^{-1}$. (f) Optical image of the pattern sample before coating with copper uniform layer. The image was trimmed to have the same scale as the reconstructed images. Scale bar on the top right shows $2 \mathrm{~mm}$.

section describes XRI's imaging sensitivity of thin films in three different analytical cases by using three different samples.

\section{A. Imaging buried layers}

$\mathrm{X}$-rays, as commonly known, can easily penetrate the sample and reach the buried layers and interfaces. By controlling the grazing incidence, one can manipulate the penetration depth of X-rays into a thin film and change electric field in-depth distribution in the sample. ${ }^{17} \mathrm{X}$-ray reflectivity is a measurement scanning grazing incidence and probing the structure of thin films in depth. By choosing specific grazing incidence (thus wavevector transfer $\mathrm{Q}_{\mathrm{z}}$ ), one can obtain a proper electric field and then emphasize specific layers or interfaces in the sample. Here, we demonstrate how we visualized a buried layer and an interface of a layered thin film in our experiment.

The sample was an ultrathin film consisting of a homogeneous copper layer and a gold pattern layer on a silicon substrate (size of the substrate: $10 \times 10 \times 0.5 \mathrm{~mm}^{3}$ ). The sample was made by an Emitech sputter coater. The layered thin film was prepared according to the following procedure: (1) the silicon substrate was put into the sputter chamber and covered by a mask made of kapton film. The mask was precut with the designed pattern: a circle containing five bars (width: $\sim 1 \mathrm{~mm}$ ) of different lengths, as shown in Fig. 3(f). Thereafter the chamber of the sputter machine was prevacuumed to $<3 \mathrm{~Pa}$ and filled with argon gas. High voltage was applied between the gold target (original material) and the substrate to ionize Ar atoms, and gold particles were then sputtered off from the target by ionized argon atoms and deposited onto the substrate. The sputtering condition for the gold layer was as follows: Ar pressure: $8 \mathrm{~Pa}$; ion current: $6 \mathrm{~mA}$; sputtering time: $60 \mathrm{~s}$. (2) The gold foil target was replaced by a copper foil target. The mask was then removed. The chamber of the sputtering machine was again pre-vacuumed to $<3 \mathrm{~Pa}$ and filled with argon gas. High voltage was applied between the copper target and the sample and the copper was sputtered onto the patterned sample. The sputtering condition for the copper layer was as follows: $\mathrm{Ar}$ pressure: $8 \mathrm{~Pa}$, ion current: $10 \mathrm{~mA}$, and sputtering time: $60 \mathrm{~s}$. The copper layer covered the gold pattern uniformly and made the gold pattern a buried layer. Reference samples were also prepared as follows: Ref. $\mathrm{Cu} / \mathrm{Au} / \mathrm{Si}$ sample was made by the same procedures (1) and (2) under the same conditions, except that no mask was applied in the procedure (1), thus sample Ref. $\mathrm{Cu} / \mathrm{Au} / \mathrm{Si}$ consists of a uniform gold layer covered by a homogeneous copper layer. Ref. $\mathrm{Cu} / \mathrm{Si}$ sample was prepared by only procedure (2), thus the Ref. $\mathrm{Cu} / \mathrm{Si}$ sample is composed only of a homogeneous copper layer on the substrate.

In order to help understand the merits and physical meaning of XRI images, the X-ray reflectivity profiles of the patterned sample and uniform reference samples were measured by an ordinary X-ray reflectometer in the laboratory, as shown in Fig. 2(b): rectangle (copper/gold/Si sample, which references the patterned part), triangle (copper/Si sample, which references the pattern-free part), and solid circle (copper/pattern gold/Si sample). To obtain the density, thickness, and roughness of the copper cover layer and that of the

TABLE I. Summary of the in-depth structure information extracted from ordinary X-ray reflectivity profiles of uniform reference samples. Superscripts $\mathrm{a}$ and $\mathrm{b}$ indicate the two different reference samples.

\begin{tabular}{lcccc}
\hline \hline Layer & Density $\left(\mathrm{g} / \mathrm{cm}^{3}\right)$ & $\mathrm{Q}_{\mathrm{c}}\left(\AA^{-1}\right)$ & Thickness $(\AA)$ & Roughness $(\AA)$ \\
\hline $\mathrm{Cu}^{\mathrm{a}, \mathrm{b}}$ & $5.17^{\mathrm{a}, \mathrm{b}}$ & $0.043^{\mathrm{a}, \mathrm{b}}$ & $172^{\mathrm{a}, \mathrm{b}}$ & $22^{\mathrm{a}, \mathrm{b}}$ \\
$\mathrm{Au}^{\mathrm{a}}$ & $19.17^{\mathrm{a}}$ & $0.078^{\mathrm{a}}$ & $212^{\mathrm{a}}$ & $11^{\mathrm{a}}$ \\
$\mathrm{Si}^{\mathrm{a}, \mathrm{b}}$ & $2.33^{\mathrm{a}, \mathrm{b}}$ & $0.032^{\mathrm{a}, \mathrm{b}}$ & $\cdots$ & $6^{\mathrm{a}, \mathrm{b}}$ \\
\hline \hline
\end{tabular}

${ }^{\mathrm{a}}$ Pattern part-Ref. $\mathrm{Au} / \mathrm{Cu} / \mathrm{Si}$.

${ }^{\mathrm{b}}$ Other part-Ref. $\mathrm{Cu} / \mathrm{Si}$. 
gold pattern layer, we fitted the $\mathrm{Q}_{\mathrm{z}}$-dependent data of the reference samples (rectangle and triangle profiles) in Fig. 2(b) using reflectivity analysis program GenX based on the Parratt formalism. ${ }^{18}$ First, the simple one layer model was used to fit the data of the copper/Si sample. Second, we applied two layer model to fit the data of the copper/gold/Si sample, while kept the same parameters for the top layer as that extracted from the copper/Si sample. Third, we assume the copper/pattern gold/Si sample has similar in-depth structures from the reference samples. The extracted in-depth structure information is given in Table I. The thickness of the copper layer is around $172 \AA$ with a roughness of $22 \AA$, while the thickness of the gold layer is $212 \AA$ with a roughness of $11 \AA$. The density of the gold layer extracted from the X-ray reflectivity is $19.17 \mathrm{~g} / \mathrm{cm}^{3}$, which is very close to the standard value $\left(19.32 \mathrm{~g} / \mathrm{cm}^{3}\right)$. However, the copper layer is a relatively low density layer with the density of around $5.17 \mathrm{~g} / \mathrm{cm}^{3}$, while the standard value of copper is $8.96 \mathrm{~g} / \mathrm{cm}^{3}$. The density of the Si substrate is $2.33 \mathrm{~g} / \mathrm{cm}^{3}$, with a roughness of $6 \AA$.

As X-ray reflectivity always assumes in-plane uniformity of samples, the measurement of X-ray reflectivity profiles from the heterogeneous sample was quite difficult. The reason could be (1) heterogeneity may complicate optical alignments; (2) the viewing area (footprint of X-rays) on the sample surface changes when we scan the grazing incident angle during the measurement, which makes the measured reflectivity a varying weighted mixture of reflectivities at different locations. In this research, the sample was positioned in the center of the viewing area, and the measured reflectivity profile: (a) is a mixture of the different lateral parts of the sample; and (b) has a different weighting factor for the lateral parts at different incidences. Fig. 2(a) is a combination of routine reflectivity scans with a position sensitive detector. The sample was fixed on the sample holder in the direction as shown in Fig. 3(f), where X-rays impinged on the sample from right and the CCD recorded the reflection projections. (The layout of the setup is displayed in Fig. 1.) The pattern structure was captured by the reflection projections of Fig. 2(a). One can see a sharp intensity drop at around $\mathrm{Q}_{\mathrm{z}}=0.045 \AA^{-1}$, which corresponds to the critical wavevector transfer $\mathrm{Q}_{c}$ of copper. The value is the same as that extracted from the conventional $\mathrm{X}$-ray reflectivity profile of the $\mathrm{Ref}$. $\mathrm{Cu} / \mathrm{Si}$ sample (nearly $\left.0.043 \AA^{-1}\right)$, considering the low $\mathrm{Q}_{\mathrm{z}}$ resolution $\left(\Delta \mathrm{Q}_{\mathrm{z}}=0.003 \AA^{-1}\right)$. Another sharp drop for some parts of the profiles is around $\mathrm{Q}_{\mathrm{z}}=0.080 \AA^{-1}$, which corresponds to the critical wavevector transfer $\mathrm{Q}_{c}$ of gold. The value equals that extracted from the conventional $\mathrm{X}$-ray reflectivity profile of the Ref. $\mathrm{Au} / \mathrm{Cu} / \mathrm{Si}$ sample (nearly $0.078 \AA^{-1}$ ). The critical wavevector transfers $\mathrm{Q}_{\mathrm{c}}$ s for each layer, as shown in Table I, are given by

$$
Q_{c}=2 k \sqrt{2 \delta}=4 \sqrt{\pi \rho r_{0}\left(1+\frac{f^{\prime}}{\mathrm{Z}}\right)},
$$

where $\mathrm{k}$ is the wavenumber $[\mathrm{k}=2 \pi / \lambda], \delta$ is the deviation of refractive index $\mathrm{n}$ from unit [related to the scattering properties of the medium], $\rho$ is the electron density, $\mathrm{r}_{0}$ is classical radius of electron, $\mathrm{f}^{\prime}$ is the dispersion correction for the element, and $\mathrm{Z}$ is the atomic number. In the high $\mathrm{Q}_{\mathrm{z}}$ range $\left(\mathrm{Q}_{\mathrm{z}}=0.08-0.14 \AA^{-1}\right)$, the profiles show ripple structures at the pattern positions. These ripples originate from thickness differences of the pattern, as will be discussed later in the $\mathrm{X}$-ray reflectivity images.

In the X-ray reflectivity imaging experiment, by looking at the X-ray reflectivity profiles in Fig. 2, five different $\mathrm{Q}_{\mathrm{z}} \mathrm{s}$ (grazing incidence was changed in the experiment, with $\mathrm{X}$-rays wavelength fixed) were chosen to emphasize different in-depth structures in the sample. 36 reflection projections (views) were measured to reconstruct the X-ray reflectivity image, which is enough to resolve the pattern $(1 \mathrm{~mm})$. Exposure time for each projection is $1 \mathrm{~s}$. Thereby, the total measuring time for 1 image is $36 \mathrm{~s}$. Figs. 3(a)-3(e) are the reconstructed images from the experiment at different wavevector transfers $\mathrm{Q}_{\mathrm{z}} \mathrm{s}$. The images were reconstructed by the homemade software utilizing the convolution backprojection algorithm. The cutoff frequency was set as 0.2 to compensate for the sparse views.

Fig. 3(a) shows the reconstructed XRI image at $\mathrm{Q}_{\mathrm{z}}=0.024 \AA^{-1}$, which is in the region of total external reflection for the copper layer, as the $\mathrm{Q}_{\mathrm{c}}$ of the top copper layer is $0.043 \AA^{-1}$. At this $\mathrm{Q}_{\mathrm{z}}$, there is only an evanescent wave propagating just several angstroms below the surface, which means the imaging is very surface sensitive at this $\mathrm{Q}_{\mathrm{z}}$. At this $\mathrm{Q}_{\mathrm{z}}, \mathrm{X}$-rays are totally reflected, and as we deposited a uniform copper layer, the image shows homogeneous contrast. Imaging at this wavevector transfer could be used to detect the surface structure such as roughness and top layer in-plane density differences.

Fig. 3(b) shows the reconstructed XRI image at $\mathrm{Q}_{\mathrm{z}}=0.049 \AA^{-1}$, which is just beyond the $\mathrm{Q}_{\mathrm{c}}$ of the copper layer and far below the $\mathrm{Q}_{\mathrm{c}}$ of the gold layer $\left(0.078 \AA^{-1}\right)$. We can see a weak contrast of the pattern (or the buried gold layer) with other parts. At this $\mathrm{Q}_{\mathrm{z}}$, there are differences between the pattern part and other parts: (a) in the pattern region, a specific fraction (depends on the $\mathrm{Q}_{\mathrm{z}}$ and surface properties of the top layer) of X-rays penetrate the top copper layer and reach the gold pattern surface. Such X-rays reaching the copper/gold interface are totally reflected by the gold. Other fractions of X-rays are reflected by the top surface, thus the reflectivity is still unity if we ignore the small fraction of absorption. (b) In the other pattern-free region, a small fraction of X-rays penetrate the top copper layer and reach the silicon surface and are weakly reflected $\left(\mathrm{Q}_{\mathrm{c}}\right.$ of $\mathrm{Si}$ is $0.032 \AA^{-1}$ ). Only that part reflected by the top copper surface contributes to the intensity of reflectivity, thus the reflectivity is smaller than unity, as shown by triangles' profile in Fig. 2 (b). Imaging at this wavevector transfer can be applied to detect the structure of the first layer such as its local thickness differences.

Figs. 3(c) and 3(d) display the reconstructed XRI images at $\mathrm{Q}_{\mathrm{z}}=0.057 \AA^{-1}$ and $\mathrm{Q}_{\mathrm{z}}=0.073 \AA^{-1}$, which are two wavevector transfers in the region between $\mathrm{Q}_{\mathrm{c}}$ of the copper layer and $\mathrm{Q}_{\mathrm{c}}$ of the buried gold layer. In this region, the reflectivity from copper dropped to $10^{-2}$ while that from the copper/ gold layer is still close to unity. This region corresponds to the 1st fringes region of the copper layer, and the reflectivity changes sinusoidally. Thereby, the two images at 
(a) Reflection Projection $\mathrm{R}\left(Q_{z}\right)$ Map

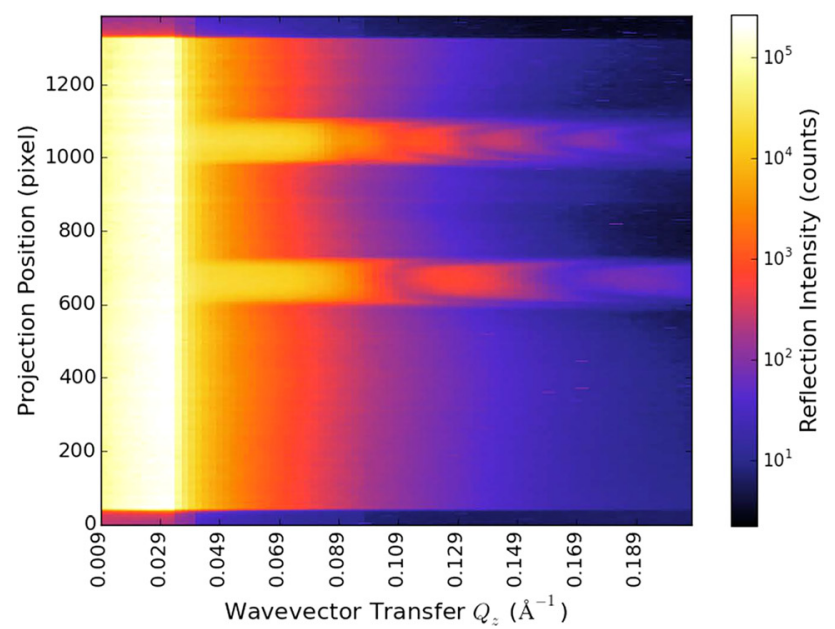

(b)

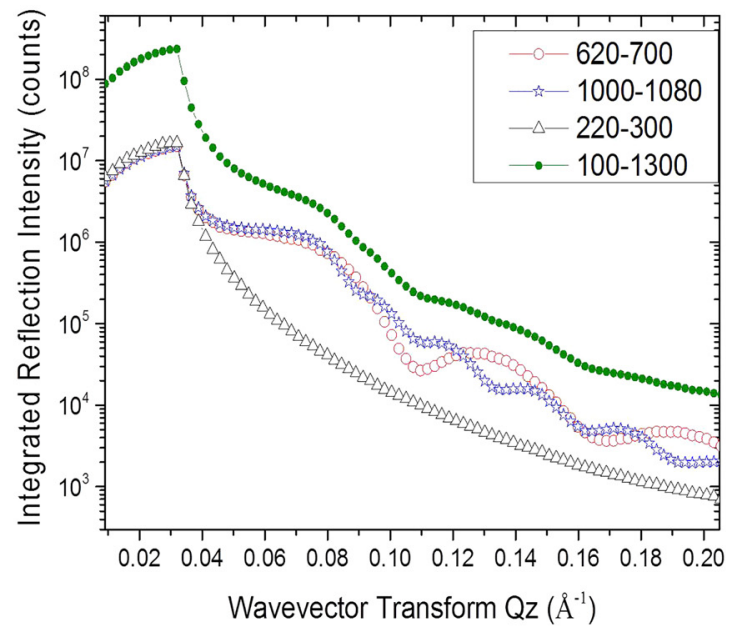

FIG. 4. X-ray reflection profiles of the gold pattern (two islands)/Si sample: two islands' thicknesses are $168 \AA$ and $84 \AA$. (a) Local reflectivity profiles of the pattern sample measured at the SR facility as the reflection projection map of the wavevector transfer $\mathrm{Q}_{\mathrm{z}}$. The scanning step for the measurement is $\Delta \mathrm{Q}_{\mathrm{z}}=0.002 \AA^{-1}$, and the data are plotted in logarithmic color scale. The sample was placed at the center of the sample chuck to ensure that the two islands were always in the irradiation area during the $\theta / 2 \theta$ scan. (b) Extracted X-ray reflectivity profiles integrated in the specific pixel range (see left figure $\mathrm{y}$-axis): middle island [620-700] (circle); top island [1000-1080] (star); bare Si [220-300] (triangle); all over the sample surface [100-1300] (solid circle).

$\mathrm{Q}_{\mathrm{z}}=0.057 \AA^{-1}$ and $\mathrm{Q}_{\mathrm{z}}=0.073 \AA^{-1}$ show different contrasts. At these wavevector transfers, the copper/gold interface is strongly enhanced, and imaging at such wavevector transfers can effectively probe the interface properties.

Fig. 3(e) shows the reconstructed XRI image at $\mathrm{Q}_{\mathrm{z}}=0.098 \AA^{-1}$, which is beyond $\mathrm{Q}_{\mathrm{c}}$ of the gold layer. At this wavevector transfer, (a) in the pattern region, X-rays reaching the gold pattern surface can also penetrate the gold layer. Such X-rays are multi-reflected weakly by the gold/silicon interface and the gold/copper interface, interfering with each other. ${ }^{19}$ The interference pattern is related to the gold layer thickness. (b) In the other pattern-free region, part of the $\mathrm{X}$-rays penetrate the top copper layer and reach the silicon surface, and are weakly reflected $\left(\mathrm{Q}_{\mathrm{c}}\right.$ of $\mathrm{Si}$ is $\left.0.032 \AA^{-1}\right)$. Although we assume that the gold film has the same thickness $(212 \AA)$ at different in-plane positions, the actual thickness of the pattern differs from position to position. In the reconstructed image, the patterns have a bright halo structure, which corresponds to the difference in thickness inside the gold film. At this $\mathrm{Q}_{\mathrm{z}}$, that ring structure is enhanced because reflectivity is the peak at the ring positions, while the adjacent black valley is related to roughly the dip position in the reflectivity curve at this area. Imaging at such wavevector transfers can be applied to detect differences in the buried layer's structure such as the roughness and the thickness. Further clarifications of the thickness contrast (shift of interference fringes influencing the image contrast) are discussed in Section III B.

\section{B. Distinguishing different thicknesses}

Ordinary surface characterization techniques only have the ability to image the morphology of the surface, and they cannot probe far into the sample. If the sample has in-plane thickness variation, the surface characterization technique usually needs to go to the edge of the object to ascertain an absolute value. Sometimes, the thickness of the film changes gradually without a sharp edge, and in such cases, it is difficult to know the thickness difference. X-ray reflectivity imaging could give a solution to this problem as X-rays could penetrate the sample and reach the buried interface. This section describes the XRI's imaging sensitivity of different layer thicknesses inside thin films.

The sample was an ultrathin film which consists of two gold isolated islands (Diameter: $0.5 \mathrm{~mm}$ ) of different thicknesses on a silicon substrate. The sample was made by the Emitech sputter coater. The layer consisting of two gold isolated islands was prepared using the following procedure: (1) the silicon substrate was set into the sputter chamber and covered by a mask made of kapton film. The mask was pre-cut with the designed pattern: one pinhole (diameter: $0.5 \mathrm{~mm}$ ). The chamber of the sputter machine was pre-vacuumed to $<3 \mathrm{~Pa}$ and filled with argon gas. High voltage was applied between the gold target (original material) and the substrate to ionize Ar atoms, and gold was then deposited onto the sample substrate. The sputtering condition was as follows: Ar pressure: $10 \mathrm{~Pa}$, ion current: $6 \mathrm{~mA}$, and sputtering time: $60 \mathrm{~s}$. (2) The mask was then shifted $1-2 \mathrm{~mm}$ to one direction. (The mask was still covering the whole area of the substrate.) The chamber of the sputter machine was pre-vacuumed again to $<3 \mathrm{~Pa}$ and filled with argon gas. High voltage was applied and the sputtering condition for the second island was as follows: Ar pressure: $10 \mathrm{~Pa}$, ion current: $6 \mathrm{~mA}$, and sputtering time: 120 s. Thus, two laterally identical islands with different thicknesses were deposited on the silicon substrate.

It is obvious that reference samples are not necessarily needed for such a simple pattern. We measured the reflectivity of these two gold isolated islands separately by $\theta / 2 \theta$ scan and the position sensitive detector (CCD camera), as shown in Fig. 4(a). It is necessary to note that the footprint length 
(L) along the X-rays forward direction on the sample is directly related to the incident angle and the beam width (w)

$$
L=w / \sin \theta,
$$

where $\mathrm{L}$ is the length of the footprint on the sample, $\mathrm{w}$ is the horizontal width of X-ray beam ( $50 \mu \mathrm{m}$ in this experiment), and $\theta$ is the incident angle of $\mathrm{X}$-rays. Although at the low $\mathrm{Q}_{\mathrm{z}}$ region (viz., low incident angle $\theta, \mathrm{Q}_{\mathrm{z}}<0.032 \AA^{-1}$, the $\mathrm{Q}_{\mathrm{c}}$ of $\mathrm{Si}$ ), the footprint of incident $\mathrm{X}$-rays is relatively large and the reflection projections include information of the reflectivities from the $\mathrm{Si}$, the reflection intensities are almost $100 \%$ from the gold film at the high $\mathrm{Q}_{\mathrm{z}}$ region. We can see a sharp intensity drop at around $\mathrm{Q}_{\mathrm{z}}=0.032 \AA^{-1}$ in Fig. 4(a), which corresponds to the critical wavevector transfer $Q_{c}$ of silicon, showing the contribution of the reflectivities from the silicon substrate at the low $\mathrm{Q}_{\mathrm{z}}$ region $\left[\mathrm{Q}_{\mathrm{z}}<0.032 \AA^{-1}\right]$. Another obvious intensity drop is at the two isolated islands positions for $\mathrm{Q}_{\mathrm{z}}=0.080 \AA^{-1}$, which corresponds to the critical wavevector transfer $Q_{c}$ of gold. Fig. 4(b) shows the reflectivity profiles integrating specific pixels range from Fig. 4(a): middle island [620-700] (circle), top island [1000-1080] (star), bare Si [220-300] (triangle), and all over the sample surface [100-1300] (solid circle). It is necessary to point out that only the reflectivity profile of bare $\mathrm{Si}$ is a pure profile from $\mathrm{Si}$, while other profiles are all mixtures of the reflectivities from gold and silicon. The reflectivity profiles including the middle and top island have two plateaus: $0.009 \AA^{-1}-0.032 \AA^{-1}$ and $0.04 \AA^{-1}-0.08 \AA^{-1}$, which correspond to the total reflection region of a silicon substrate and a gold film, as the profiles are the mixture of the gold layer and the silicon substrate.

We conducted a simple calculation to see the mixture ratio of gold to silicon in the area irradiated by the incident $\mathrm{X}$-ray beam

$$
\frac{S_{\text {gold }}}{S_{\text {total }}}=\frac{R_{\text {total }}\left(Q_{z 1}\right)-R_{\text {silicon }}\left(Q_{z 1}\right)}{R_{\text {total }}\left(Q_{z 2}\right)} \div \frac{w_{\text {accept }}\left(Q_{z 1}\right)}{w_{\text {accept }}\left(Q_{z 2}\right)},
$$

where $S_{\text {gold }}$ is the surface area of the gold island, $S_{\text {total }}$ is the surface area of $\mathrm{X}$-rays footprint in the pixel range (e.g., 1000-1080), $\mathrm{R}_{\text {total }}\left(\mathrm{Q}_{\mathrm{z}}\right)$ and $\mathrm{R}_{\text {silicon }}\left(\mathrm{Q}_{\mathrm{z}}\right)$ are the reflection intensity from the gold included area and bare silicon area at a specific $Q_{z}$, respectively, and $\mathrm{w}_{\mathrm{accept}}\left(\mathrm{Q}_{\mathrm{z}}\right)$ is the accepted horizontal width of X-rays. [As the $\mathrm{Q}_{z}$ s chosen in this calculation are small enough that the $\mathrm{X}$-rays footprint is always larger than the sample size $(10 \mathrm{~mm})$, the accepted X-rays (width) is linearly related to $\sin \theta$ according to Eq. (5).] The ratio can be calculated with Eq. (6) by looking up the reflection intensities at $\mathrm{Q}_{\mathrm{z}}=0.0319 \AA^{-1}$ and at $0.0638 \AA^{-1}$ in Fig. 4(b)

$$
\begin{aligned}
\mathrm{S}_{\text {gold }} / \mathrm{S}_{\text {total }}= & \left(1.36 \times 10^{6}-1.10 \times 10^{5}\right) \div\left(1.51 \times 10^{7}\right) \\
& \div 2=4.14 \%,
\end{aligned}
$$

where $1.36 \times 10^{6}$ is the reflection intensity from the mixture area $\{[(1080-1000) \times 6.45 \mu \mathrm{m}] \times 10 \mathrm{~mm}\}$ at $\mathrm{Q}_{\mathrm{z}}=0.0638 \AA^{-1}$, $1.10 \times 10^{5}$ is the reflection intensity from pure silicon $\{[(300-$ $220) \times 6.45 \mu \mathrm{m}] \times 10 \mathrm{~mm}\}$ at $\mathrm{Q}_{\mathrm{z}}=0.0638 \AA^{-1}$, and $1.51 \times 10^{7}$ is the reflection intensity from the mixture area $\{[(1080-1000)$ $\times 6.45 \mu \mathrm{m}] \times 10 \mathrm{~mm}\}$ at $\mathrm{Q}_{\mathrm{z}}=0.0319 \AA^{-1}$. In order to compare the reflection intensities, we need to consider the accepted $\mathrm{X}$-rays intensity and include the weighting factor $\mathrm{w}_{\text {accept }}$ $\left(\mathrm{Q}_{\mathrm{z}}=0.0638 \AA^{-1}\right) / \mathrm{w}_{\text {accept }} \quad\left(\mathrm{Q}_{\mathrm{z}}=0.0319 \AA^{-1}\right)=2$. Assuming the perfect round area of the gold island, this calculation implies that the diameter of the gold island is $2 \times$ $\sqrt{[10 \times(80 \times 0.00645) \times 4.14 \%] / \pi}=0.52 \mathrm{~mm}$, which is quite close to the designed value.

The thickness of the middle island extracted from the reflectivity profile in Fig. 4(b) is around $83 \AA$, and the thickness of the top island is $180 \AA$, which is around twice that of the middle island (related to the doubled deposition time in the sample preparation procedure). Since the X-rays viewing area and the weighting factors for the mixture of gold and silicon are different at different incident angle, we only fitted the interference fringes position [only influenced by the gold film] of the $Q_{z}$-dependent data in Fig. 4(b) and extracted the thicknesses of the gold patterns. In the high $\mathrm{Q}_{\mathrm{z}}$ range $\left(Q_{z}=0.08-0.20 \AA^{-1}\right)$, the reflection projection also shows ripple structures at the pattern positions. As no additional cover layer was presented, these ripple structures are obviously due to the thickness differences in each gold island. We sliced at each pixel in Fig. 4(a) and plotted many X-ray reflectivity profiles. Although the footprint of incident $\mathrm{X}$-rays always covers the whole pattern, we fitted the interference fringes position of those reflectivity profiles and extracted the semi-local thicknesses [averaged along X-rays forward direction] in the gold island. The thicknesses of each slice labeled by each pixel value (p) of the middle island in Fig. 4(a) are summarized in Table II. At the edge of the island $(p=620)$, the film is thinner $(76 \AA)$. When approaching the center of the island, the thickness increases. The uniform plateau has the thickest value ( $88 \AA$ ). According to the analysis, the island is made of a uniform thickness plateau (650-690) near the center and a slope (620-640) at the edge. As mentioned above, the values in Table II are still averaged values-averaging along the X-rays forward direction. That is one reason why we urgently need the present technique to obtain a $2 \mathrm{D}$ image of reflectivity. The top island has a similar structure, and here, we omit the discussion.

For the gold islands sample, we also chose five different $\mathrm{Q}_{\mathrm{Z}} \mathrm{s}$ (grazing incidence was changed in the experiment, with $\mathrm{X}$-rays wavelength fixed) to emphasize different in-depth structure in the sample. 72 reflection projections (views) were measured to reconstruct the $\mathrm{X}$-ray reflectivity image, which is enough to resolve the pattern $(0.5 \mathrm{~mm})$. The total measuring time for 1 image was 72 s. Figs. 5(a)-5(e) are the reconstructed images from the experiment at different wave-

\begin{tabular}{|c|c|c|c|c|}
\hline Pixel position & 620 & 630 & 640 & 650 \\
\hline Thickness (̊) & 76 & 80 & 82 & 86 \\
\hline Pixel position & \multicolumn{2}{|c|}{$660-680$} & 690 & 700 \\
\hline Thickness (̊) & \multicolumn{2}{|c|}{88} & 87 & 85 \\
\hline
\end{tabular}
vector transfers $\mathrm{Q}_{\mathrm{z}} \mathrm{s}$. The reconstruction algorithm used here

TABLE II. Summary of thicknesses of each slice labeled by each pixel value of the middle gold island in Fig. 4(a). 

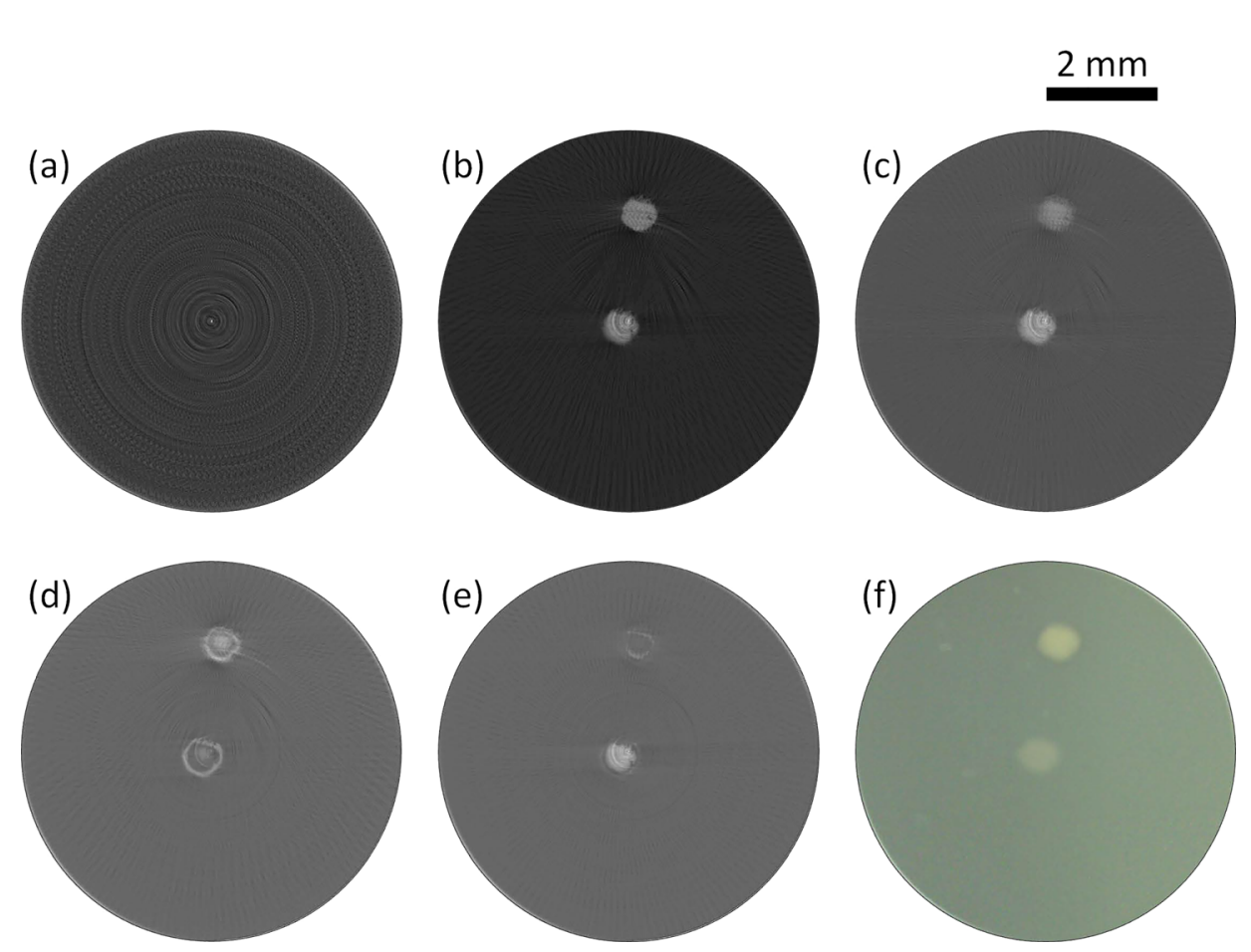

FIG. 5. Reconstructed X-ray reflectivity images of the gold (two islands with different thicknesses)/Si sample at different wavevector transfers. Parameters for image reconstruction: 72 views, cutoff frequency for Ram-Lak filter: 0.5 . (a) $\mathrm{Q}_{\mathrm{z}}=0.030 \AA^{-1}$; (b) $\mathrm{Q}_{\mathrm{z}}=0.068 \AA^{-1}$; (c) $\mathrm{Q}_{\mathrm{z}}=0.089 \AA^{-1}$; (d) $\mathrm{Q}_{\mathrm{z}}=0.110 \AA^{-1}$; (e) $\mathrm{Q}_{\mathrm{z}}=0.132 \AA^{-1}$. (f) Optical image of the two island pattern. The image was trimmed to have the same scale as the reconstructed images. Scale bar on the top right shows $2 \mathrm{~mm}$.

was also the convolution back-projection algorithm with the cutoff frequency 0.5 .

Fig. 5(a) shows the reconstructed XRI image at $\mathrm{Q}_{\mathrm{z}}=0.030 \AA^{-1}$, which is in the region of total external reflection for both silicon and gold, as $\mathrm{Q}_{\mathrm{c}}$ of silicon and gold are $0.032 \AA^{-1}$ and $0.078 \AA^{-1}$, respectively. At this $\mathrm{Q}_{\mathrm{z}}$, the reflectivities from the silicon substrate and gold islands are both unity, so there is no contrast between the pattern and the other pattern-free part in the image. At this $\mathrm{Q}_{\mathrm{z}}, \mathrm{X}$-rays are totally reflected, and the image shows homogeneous contrast.

Fig. 5(b) shows the reconstructed XRI image at $\mathrm{Q}_{\mathrm{z}}=0.068 \AA^{-1}$, which is between the $\mathrm{Q}_{\mathrm{c}}$ of gold $\left(0.078 \AA^{-1}\right)$ and $\mathrm{Q}_{\mathrm{c}}$ of silicon $\left(0.032 \AA^{-1}\right)$. We can see a strong contrast between the gold islands and the silicon substrate. At this $\mathrm{Q}_{\mathrm{z}}$, in the pattern region, $\mathrm{X}$-rays are totally reflected by the gold and the reflectivity is unity. In the silicon part, X-rays are weakly reflected $\left(\mathrm{Q}_{\mathrm{c}}\right.$ of $\mathrm{Si}$ is $\left.0.032 \AA^{-1}\right)$. The two gold islands show the same contrast, as X-rays do not penetrate the gold island film at this $\mathrm{Q}_{\mathrm{z}}$. Imaging at this wavevector transfer can be used to detect thin film's surface properties such as the shape and the roughness of the islands' surface.

Fig. 5(c) shows the reconstructed XRI image at $\mathrm{Q}_{\mathrm{z}}=0.088 \AA^{-1}$, which is beyond the $\mathrm{Q}_{\mathrm{c}}$ of gold $\left(0.078 \AA^{-1}\right)$. We can see a contrast between the middle and the top gold (a) Reflection Projection $\mathrm{R}\left(Q_{z}\right)$ Map

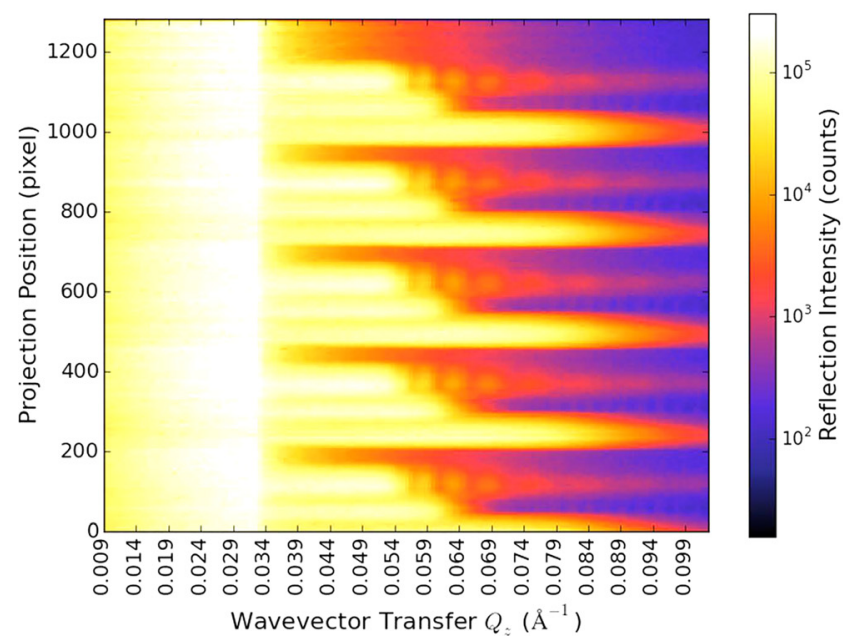

(b)

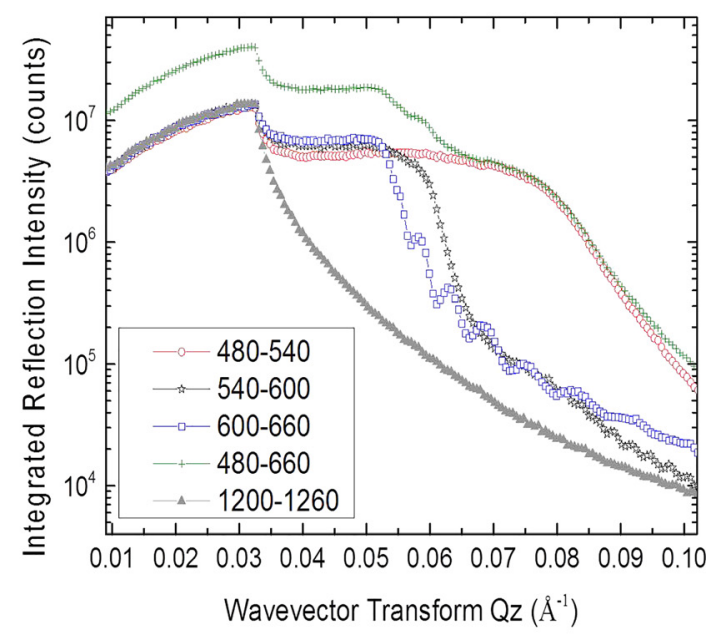

FIG. 6. X-ray reflection profiles of the pattern target of gold, copper, chromium line patterns/glass. Line width: 0.2 mm; film thickness: $1000 \AA$. (a) Local reflectivity profiles of the pattern sample measured at the $S R$ facility as the reflection projection map of the wavevector transfer $Q_{z}$. The scanning step for the measurement is $\Delta \mathrm{Q}_{\mathrm{z}}=0.0005 \AA^{-1}$, and the data are plotted in logarithmic colorscale. The sample was positioned to ensure the line patterns parallel to the X-ray forward direction. (b) Extracted X-ray reflectivity profiles integrated in the specific pixel range (see left figure $y$-axis): gold area [480-540] (circle), copper part [540-600] (star), chromium [600-660] (rectangle). Area includes all [480-660] (cross), and bare Si [1200-1260] (solid triangle). 
island, in addition to the contrast between the gold islands and the silicon substrate. At this wavevector transfer, the image of the middle island is brighter than that of the top island, which means higher reflectivity from the middle island, as shown in Fig. 4(b). In both islands, X-rays penetrate the gold films. The difference is that the reflectivity from the thick top island deviates more from unity than that of the thin middle island. Imaging at this wavevector transfer can be used to detect layer properties such as the shape and the thickness of the thin film. Fig. 5(d) shows the reconstructed XRI image at $\mathrm{Q}_{\mathrm{z}}=0.010 \AA^{-1}$, which is in the range of interference fringes region. At this wavevector transfer, the image of the top island is brighter than that of the middle island, which means higher reflectivity from the top island, whose feature also coincides with that shown in Fig. 4. The image contrast was reversed compared with the image at $\mathrm{Q}_{\mathrm{z}}=0.088 \AA^{-1}$. In addition, the image is not uniform inside each island, because the local thicknesses of the island are different, as shown in Table II. The islands have complex structures: the middle island is mainly composed of a ring structure, while there are shadows inside the ring. The top island consists of an outer shell and an inner uniform structure. Fig. 5(e) shows the reconstructed XRI image at $\mathrm{Q}_{\mathrm{z}}=0.013 \AA^{-1}$. The image contrast is reversed again compared with the image at $\mathrm{Q}_{\mathrm{z}}=0.010 \AA^{-1}$. At this wavevector transfer, the image at the middle island position is brighter than that of the top island, which means higher reflectivity from the middle island. These features also accord with that shown in Fig. 4. At this $\mathrm{Q}_{\mathrm{z}}$, the substructures inside each island are different from the previous XRI image: the image of the middle island is now mainly composed of a uniform plateau and a dark ring. However, in case of the top island, only the ring structure is still being resolved. By combining imaging at different wavevector transfers in the interference fringes region, one can visualize not only obvious differences (e.g., differences between the middle and the top island), but also very tiny structure differences in sub $\mathrm{nm}$ scale (e.g., substructure of the film near the edge of each island). By analyzing the image, one can recognize how the two points differ. This technique has high sensitivity along the depth, typically $\sim \AA$, while higher $Q_{z}$ could further emphasize higher sensitivity.

\section{Distinguishing different materials}

As commonly known, X-ray reflectivity is not an element selective technique. However, by introducing the in-plane spatial resolution, X-ray reflectivity imaging can provide selective ability when we carefully choose the wavevector transfer of interest. The sample used here is a commercial available patterned target: the repetition of the unit composed of gold, copper, and chromium line patterns on a glass substrate, as shown in Fig. 7(d). The sample size is $10 \times 10 \mathrm{~mm}^{2}$. The single line width for each material is $0.2 \mathrm{~mm}$ with a thickness of $1000 \AA$.

We can also measure reflectivity projections of the target depending on wavevector transfers $\mathrm{Q}_{\mathrm{z}} \mathrm{s}$. In order to obtain the reflectivity from different materials independently, the sample was aligned to make the line pattern parallel to the

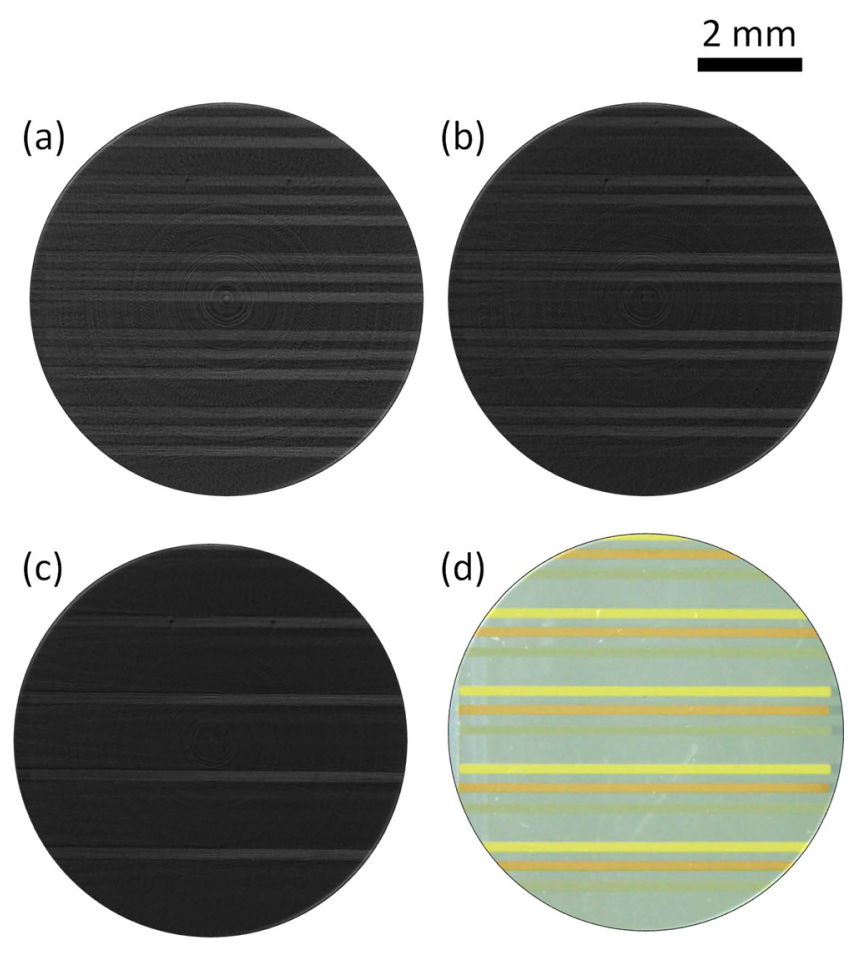

FIG. 7. Reconstructed X-ray reflectivity images of the commercial available sample of gold, copper, chromium line patterns/glass at different wavevector transfers. Parameters for image reconstruction: 72 views, cutoff frequency for Ram-Lak filter: 0.5. (a) $\mathrm{Q}_{\mathrm{z}}=0.053 \AA^{-1}$; (b) $\mathrm{Q}_{\mathrm{z}}=0.056 \AA^{-1}$; (c) $\mathrm{Q}_{\mathrm{z}}=0.063 \AA^{-1}$. Three reconstructed images have been rotated $3^{\circ} \mathrm{CW}$ for comparison. (d) Optical image of the sample. The image was trimmed to have the same scale as the reconstructed images. Scale bar on the top right shows $2 \mathrm{~mm}$.

X-rays forward direction. The $\mathrm{Q}_{\mathrm{c}} \mathrm{s}$ of chromium, copper, and gold are $0.053 \AA^{-1}, 0.057 \AA^{-1}$, and $0.079 \AA^{-1}$, respectively, as shown in Fig. 6(a). Such values are quite close to the standard values. Fig. 6(b) shows extracted reflection intensity profiles integrated in the specific pixels range: gold area [480-540] (circle), copper part [540-600] (star), chromium [600-660] (rectangle), area that includes all [480-660] (cross), and bare $\mathrm{SiO}_{2}$ [1200-1260] (solid triangle). Although the sample was aligned, it was still difficult to make the line pattern perfectly parallel to the beam. The reflectivity profiles are influenced by such a tilt $\left[3^{\circ} \mathrm{CCW}\right.$ direction, shown in the caption of Fig. 7(d)]. This makes the reflectivity profile a mixture of neighboring areas. All materials, gold, copper, and chromium reflectivity profiles, possess a drop at $\mathrm{Q}_{\mathrm{z}}=0.032 \AA^{-1}$, which is the $\mathrm{Q}_{\mathrm{c}}$ of the glass substrate. In the displayed $\mathrm{Q}_{\mathrm{z}}$ range, gold does not have the interference fringes part. Chromium shows interference fringes in the high $\mathrm{Q}_{\mathrm{z}}$ part, while copper's fringes are smeared because of the overlap with neighboring gold and chromium.

We chose three different $\mathrm{Q}_{\mathrm{z}}$ s to selectively image different materials. 72 reflection projections (views) were measured to reconstruct the $\mathrm{X}$-ray reflectivity image, which is enough to resolve the pattern $(0.2 \mathrm{~mm})$. The total measuring time for 1 image is $72 \mathrm{~s}$. Figs. 7(a)-7(c) are the reconstructed images from the experiment at different wavevector transfers $\mathrm{Q}_{\mathrm{Z}} \mathrm{s}$. The images were reconstructed utilizing the same convolution back-projection algorithm with the cutoff frequency 0.5 . 
Fig. 7(a) shows the reconstructed XRI image at $\mathrm{Q}_{\mathrm{z}}=0.050 \AA^{-1}$, which is below the $\mathrm{Q}_{\mathrm{c}}$ of all materials but above the $\mathrm{Q}_{\mathrm{c}}$ of glass $\left(0.032 \AA^{-1}\right)$. We can see a contrast of three line patterns on the black substrate. X-rays that reach the surface of each material are totally reflected by the surfaces, while those that reach the glass substrate strongly penetrate the bulk and are weakly reflected. Imaging at this wavevector transfer shows the pattern of all three materials.

Fig. 7(b) shows the reconstructed XRI image at $\mathrm{Q}_{\mathrm{z}}=0.056 \AA^{-1}$, which is below the $\mathrm{Q}_{\mathrm{c}}$ of gold and copper but above the $\mathrm{Q}_{\mathrm{c}}$ of chromium $\left(0.053 \AA^{-1}\right)$. We can resolve the contrast of two line patterns on the black substrate, and the bottom line is not resolved. At this $\mathrm{Q}_{\mathrm{z}}, \mathrm{X}$-rays that reach the surfaces of gold and copper are totally reflected, while those that reach the surface of chromium are weakly reflected and the reflectivity from the chromium surface is much less than unity. Imaging at this wavevector transfer shows the pattern of the two materials with higher electron densities.

Fig. 7(c) shows the reconstructed XRI image at $\mathrm{Q}_{\mathrm{z}}=0.060 \AA^{-1}$, which is below the $\mathrm{Q}_{\mathrm{c}}$ of gold but above the $\mathrm{Q}_{\mathrm{c}}$ of chromium $\left(0.053 \AA^{-1}\right)$ and copper $\left(0.057 \AA^{-1}\right)$. We can see a contrast of one line patterns on the black substrate, and the two bottom lines are not resolved. At this $\mathrm{Q}_{\mathrm{z}}$, only $\mathrm{X}$-rays that reach the surface of gold are strongly reflected. Imaging at this wavevector transfer shows only one material pattern, viz., only gold is selected in the image.

Three different materials were selected one by one using this imaging technique, as discussed in the paragraphs above. However, it is necessary to emphasize that this technique does not give spectroscopic element information like characteristic energies in the X-ray fluorescence technique, but probes the electron densities of the sample. The critical wavevector transfer $\mathrm{Q}_{\mathrm{c}}$ is decided by Eq. (4): the $\mathrm{Q}_{\mathrm{c}}$ is proportional to the square root of density $\rho$, hence the sensitivity of XRI to density is mediocre. The three different materials have different electron densities, so the critical wavevector transfer $\mathrm{Q}_{\mathrm{c}} \mathrm{s}$ get different values. This imaging technique can selectively visualize different materials by taking advantage of the different total reflection plateau regions.

\section{In-plane spatial resolution}

In this experiment, the vertical angular divergence of the X-ray beam is nearly $0.02 \mathrm{mrad}$, and the distance between the sample and the detector is around $300 \mathrm{~mm}$, so the in-plane spatial resolution of this technique is limited only by the pixel size of the CCD camera: $6.45 \mu \mathrm{m}$ $(6.45 \mu \mathrm{m}>0.02 \times 300 \mu \mathrm{m})$. In practice, the optical lens for the scintillation layer may smear the image and the spatial resolution may also be affected by the imperfection of optics. The theoretical predicted value is around $15 \mu \mathrm{m}$. A 1951 US Air Force (USAF) resolution test target was used for directly checking the in-plane spatial resolution, and it includes a pattern of different sized horizontal and vertical $\mathrm{Cr}$ bars on a glass substrate. The resolution target was cut to an appropriate size to obtain the X-ray reflectivity images. Fig. 8(c) shows the optical microscopy image of the resolution target.
We chose $Q_{z}=0.046 \AA^{-1}$ to enhance the contrast between chromium $\left(\mathrm{Q}_{\mathrm{c}}=0.053 \AA^{-1}\right)$ and the glass substrate $\left(\mathrm{Q}_{\mathrm{c}}=0.032 \AA^{-1}\right) .360$ reflection projections (views) were measured to obtain the X-ray reflectivity image, in order to resolve the finer structure. The total measuring time was 360 s. Fig. 8(a) shows the reconstructed image from the experiment. We reconstructed the image utilizing the convolution back-projection algorithm with the cutoff frequency set at 0.5. Fig. 8(b) shows the enlarged image to indicate the resolution, while Fig. 8(d) is the optical microscopy image in the same magnification scale. The smallest width resolved is nearly $20 \mu \mathrm{m}$, which is not far from the prediction. In Section III B, it is demonstrated that the sensitivity of XRI in the depth direction is $\sim 1 \AA$. In summary, the spatial resolutions along three orthogonal directions at the present stage are established: $20 \mu \mathrm{m} \times 20 \mu \mathrm{m} \times 0.1 \mathrm{~nm}$.

In the experiment, the specification of $\mathrm{Q}_{\mathrm{z}}$ is decided by the incident grazing angle, as shown in Eq. (1). According to the geometry of this experiment, the beam width is $0.05 \mathrm{~mm}$ $(\mathrm{H}) \times 8 \mathrm{~mm}(\mathrm{~V})$. In order to get a square viewing area (footprint in the reflectivity measurement) of $8 \mathrm{~mm}(\mathrm{H}) \times 8 \mathrm{~mm}$ (V), the incident angle can be calculated from Eq. (5) and should be $6.25 \mathrm{mrad}$, which corresponds to the Qz of $0.100 \AA^{-1}$. At different $\mathrm{Q}_{\mathrm{z}} \mathrm{s}$, the width of the incident beam (the width of entrance slit) should have been adjusted to a proper value as suggested in Eq. (5). In our experiment, we applied a slit with a fixed width of $0.05 \mathrm{~mm}$, and it may have caused some distortions in the images. However, these distortions still do not influence the previous data analysis (large pattern), although it may smear the images and

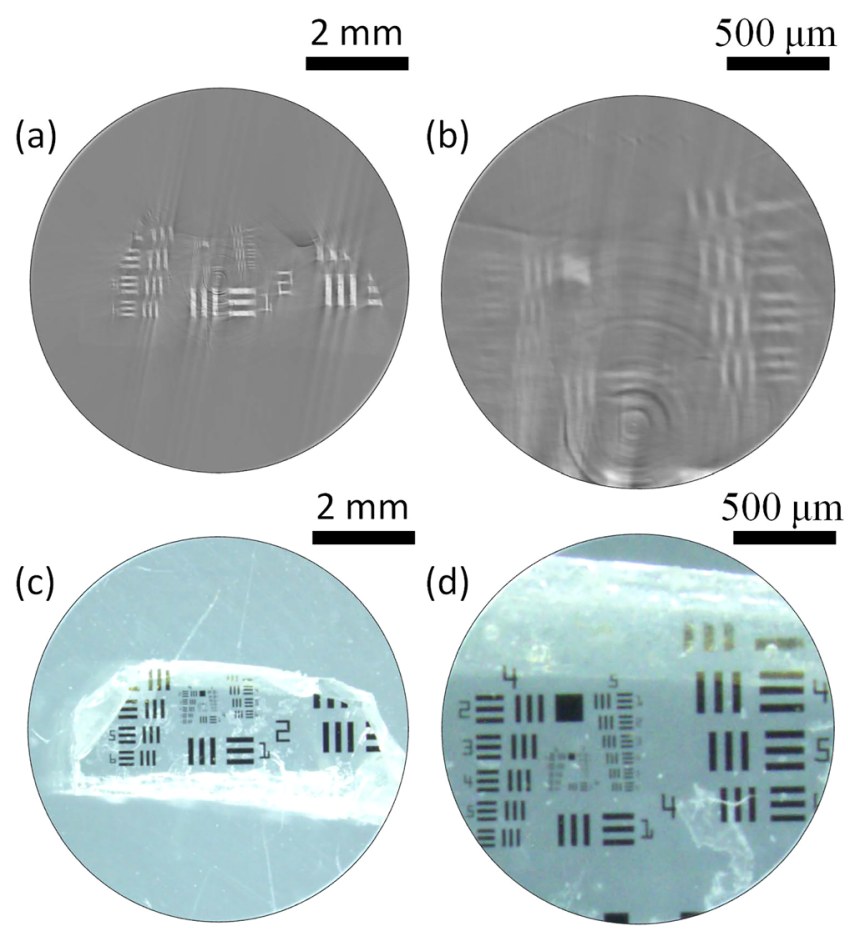

FIG. 8. Reconstructed X-ray reflectivity images and optical images of the USAF resolution target in two different magnifications. The resolution target was cut to an appropriate size suitable for the viewing area. (a) Low magnification and (b) high magnification of the proper reconstructed image; (c) optical image and (d) enlarged image of the interested area corresponding to the same scale as (a) and (b). Scale bars are shown at the top right. 
influence the spatial resolution. When applied to more difficult cases such as the observation of structures approaching the spatial resolution limit or the detection of extremely tiny differences, such distortions or smearing effects should be taken into account. In such cases, introducing a width adjustable slit is helpful.

\section{CONCLUSION}

In this work, we have successfully visualized the buried layers and interfaces in ultrathin films by a novel X-ray reflectivity imaging technique using synchrotron radiation. By collecting a series of reflection projection intensity profiles during an in-plane rotational scan of the sample, under grazing-incidence reflection geometry, we can obtain the $\mathrm{X}$-ray reflectivity image by employing mathematical image reconstruction. By tuning the grazing angle, we successfully obtained different image contrasts, which indicate that different in-depth structures were enhanced. The different X-ray reflectivities at local in-plane areas of thin films at a specific wavevector transfer show different in-plane structures of the sample. By comparing different images at different incident angles, we not only visualized buried interfaces but also obtained nice contrasts in tiny thickness differences (in the order of $\sim \AA$ ) and selectively visualized different materials with different densities. We also confirmed that the in-plane spatial resolution is better than $20 \mu \mathrm{m}$. This technique provides high in-depth sensitivity $(\sim \AA)$ with quite good spatial resolution $(20 \mu \mathrm{m})$.

\section{ACKNOWLEDGMENTS}

The authors would like to thank Dr. M. Mizusawa for her useful discussion and assistance during the present work.
This work was done with the approval of the Photon Factory Program Advisory Committee (Proposal No. 2015G053).

${ }^{1}$ K. Sakurai, M. Mizusawa, M. Ishii, S. Kobayashi, and Y. Imai, J. Phys.: Conf. Ser. 83, 012001 (2007).

${ }^{2}$ P. Fenter, C. Park, Z. Zhang, and S. Wang, Nat. Phys. 2, 700 (2006)

${ }^{3}$ P. Fenter, S. S. Lee, Z. Zhang, and N. C. Sturchio, J. Appl. Phys. 110, 102211 (2011).

${ }^{4}$ P. Fenter, C. Park, V. Kohli, and Z. Zhang, J. Synchrotron Radiat. 15, 558 (2008).

${ }^{5}$ M. J. Highland and P. Fenter, J. Synchrotron Radiat. 21, 1252 (2014).

${ }^{6}$ S. Roy, D. Parks, K. A. Seu, R. Su, J. J. Turner, W. Chao, H. E. Anderson, S. Cabrini, and S. D. Kevan, Nat. Photonics 5, 243 (2011).

${ }^{7}$ T. Sun, Z. Jiang, J. Strzalka, L. Ocola, and J. Wang, Nat. Photonics 6, 586 (2012).

${ }^{8}$ C. Zhu, R. Harder, A. Diaz, V. Komanicky, A. Barbour, R. Xu, X. Huang, M. S. Pierce, A. Menzel, H. You, C. Zhu, R. Harder, A. Diaz, V. Komanicky, A. Barbour, R. Xu, X. Huang, Y. Liu, M. S. Pierce, and A. Menzel, Appl. Phys. Lett. 106, 101604 (2015).

${ }^{9}$ V. A. Innis-Samson, M. Mizusawa, and K. Sakurai, Anal. Chem. 83, 7600 (2011).

${ }^{10}$ V. A. Innis-Samson, M. Mizusawa, and K. Sakurai, Adv. X-Ray Chem. Anal., Jpn. 43, 391 (2012).

${ }^{11}$ J. Jiang and K. Sakurai, "X-ray reflectivity imager with $15 \mathrm{~W}$ power X-ray source," Rev. Sci. Instrum. (to be published).

${ }^{12}$ M. Ando, Y. Satow, H. Kawata, T. Ishikawa, P. Spieker, and S. Suzuki, Nucl. Instrum. Methods Phys. Res., Sect. A 246, 144 (1986).

${ }^{13}$ J. Daillant and A. Gibaud, X-Ray and Neutron Reflectivity: Principles and Applications, 1st ed. (Springer, Berlin, 1999).

${ }^{14} \mathrm{G}$. T. Herman, Fundamentals of Computerized Tomography: Image Reconstruction from Projections, 2nd ed. (Springer, London, 2009).

${ }^{15}$ A. C. Kak and M. Slaney, Principles of Computerized Tomographic Imaging (IEEE Press, New York, 1999).

${ }^{16} \mathrm{~F}$. Natterer, The Mathematics of Computerized Tomography (Society for Industrial and Applied Mathematics, Philadelphia, 2001).

${ }^{17}$ E. Gann, A. Watson, J. R. Tumbleston, J. Cochran, H. Yan, C. Wang, J. Seok, M. Chabinyc, and H. Ade, Phys. Rev. B 90, 245421 (2014).

${ }^{18}$ M. Björck and G. Andersson, J. Appl. Cryst. 40, 1174 (2007).

${ }^{19}$ L. G. Parratt, Phys. Rev. 95, 359 (1954). 